\title{
Robustness of the Unscented Kalman Filter for State and Parameter Estimation in an Elastic Transmission
}

\author{
Edvard Naerum ${ }^{1,2}$, H. Hawkeye King ${ }^{3}$ and Blake Hannaford ${ }^{3}$. Paper-ID 86 \\ ${ }^{1}$ The Interventional Centre, Rikshospitalet University Hospital, Oslo, Norway \\ ${ }^{2}$ Faculty of Medicine, University of Oslo, Norway \\ ${ }^{3}$ Department of Electrical Engineering, University of Washington, Seattle, WA, USA \\ E-mail: edvard.narum@medisin.uio.no, (hawkeye1, blake) du.washington.edu
}

\begin{abstract}
The Unscented Kalman Filter (UKF) was applied to state and parameter estimation of a one degree of freedom robot link with an elastic, cable-driven transmission. Only motor encoder and command torque data were used as input to the filter. The UKF was used offline for joint state and model-parameter estimation, and online for state estimation. This paper presents an analysis of the robustness of the UKF to unknown/unmodeled variation in inertia, cable tension and contact forces, using experimental data collected with the robot.

Using model parameters found offline the UKF successfully estimated motor and link angles and velocities online. Although the transmission was very stiff, and hence the motor and link states almost equal, information about the individual states was obtained. Irrespective of variation from nominal conditions the UKF link angle estimate was better than using motor position as an approximation (i.e. inelastic transmission assumption). The angle estimates were particularly robust to variation in operating conditions, velocity estimates less so. A near-linear relationship between contact forces and estimation errors suggested that contact forces might be estimated using this error information.
\end{abstract}

\section{INTRODUCTION}

\section{A. Background}

State and parameter knowledge are of prime importance in robot manipulation. Accurate control of a robot requires knowing the robot state and good controller design requires an accurate model of the robot. Sensors are often noisy so filtering is necessary to get a good state measurement. Filters, however, can introduce a phase lag and degrade performance. Model based state estimation may be a good way to obtain state estimates from noisy sensors, or a reduced set of sensors.

The BioRobotics Lab at the University of Washington has developed the RAVEN robot, a new six degree of freedom (DoF) surgical robot prototype [1]. The RAVEN is cabledriven, allowing the motors to be removed from the arms and attached to the base. This decreases the arms' inertia, but the cables introduce elasticity into the system. That, in turn, creates additional states since the link is elastically coupled to the motor. The RAVEN has encoders mounted on the motors but not on the link side, since encoders on the links themselves would require additional, complex wiring. Furthermore, the encoder signal is highly quantized making noise-free velocity estimation difficult to compute. In addition, several of the model parameters are not directly observable, namely the cable stiffness and damping, and friction. This paper focuses on a single-DoF RAVEN-like testbed to study the problems of state estimation and parameter estimation in an elastic transmission.

\section{B. Related Work}

Most published studies on elastic transmissions have focused on elastic-joint robots. For a cable-driven robot the analysis is normally more complicated due to coupling between cable runs for multiple joints. However, there is no coupling in a 1-DoF cable-driven robot, and the modeling coincides with that of a 1-DoF elastic-joint robot.When extrapolating the results of this paper back to a multi-DoF robot such as the RAVEN the coupling, which is a kinematic relationship, must be taken into account.

Research on state and parameter estimation in elastic-joint robots gained momentum in the 1980's, together with the development of controller designs that required knowledge of the robot's state and model. Nicosia and Tornambé [2] developed a method to estimate the parameters of an elasticjoint robot using the output injection technique used in observers for nonlinear systems. The state of the robot was assumed known. In [3] Nicosia et al. designed approximate state observers for elastic-joint robots, and derived conditions under which exact observers exist. Their work required some measurement of the state at both sides of the transmission. Jankovic [4] proposed a reduced order high-gain state observer for elastic-joint robots that only required measurement of the motor side state, i.e. motor angle and velocity. A variable structure observer was designed by Léchevin and Sicard in [5] for elastic-joint manipulators. Their observer used link angle measurements. Abdollahi et al. [6] built a general state observer for nonlinear systems where a neural network was used to learn the system's dynamics. The observer's potential was demonstrated with an application to elastic-joint robots with measurement of motor angle and velocity.

\section{Present Work}

The task at hand in this paper is simultaneous state and parameter estimation of an elastic transmission with only motor angle measurements available. The aforementioned studies either require the knowledge of more states, or they only allow the estimation of certain parameters, such as the transmission elasticity. We would like to estimate several parameters, including friction. Therefore, we approach the problem through straight-forward application of the Unscented Kalman Filter (UKF) [7, 8, 9]. The primary difference between the UKF and the Extended Kalman Filter (EKF) is that the $\mathrm{UKF}$ is capable of performing nonlinear estimation without 
linearization, whereas the EKF linearizes the nonlinear system and applies a regular Kalman filter algorithm. The UKF was chosen as it has been argued that it should replace the EKF in estimation applications for nonlinear systems because of its simpler implementation and better performance [7, 9]. Araki et al. [10] estimated the parameters of a robot with rigid joints using a UKF, and experiments were done with a two-DoF robot. Other works not related to robotics that employ the UKF for state and/or parameter estimation include Zhao et al. [11], Gove and Hollinger [12] and Zhan and Wan [13].

We present a study of simultaneous state and parameter estimation for offline system identification, and an online state estimator for smooth, noise-free state measurement with no phase distortion. The UKF is used in both cases. The main contribution of this work is an experimental examination of robustness of the UKF to unknown variations in inertia, cable tension and force disturbances. We are primarily interested in applications to teleoperation, and the motivating factors behind the robustness experiments are

1) to investigate the possibility of estimating contact forces with an elastic-joint robot with partial state measurement in a bilateral teleoperation setting, and

2) to get an indication of the performance of a simple jointlevel UKF for state estimation and feedback control in a multi-DoF robot.

A more general, but important question that also needs to be answered is whether, and with what accuracy we can estimate the link state using motor angle measurements. In other words, can we infer better information about the actual link state by the application of an advanced filter like the UKF, or will using the motor state as an approximation to the link state be just as useful, i.e. a rigid robot assumption?

\section{System \& Modeling}

\section{A. Hardware}

To test the UKF robustness in a real world setting, a simple 1-DoF test platform was employed (see Fig. 1). Termed the "pulleyboard" it uses all the same hardware as the RAVEN surgical robot, and is intended as a testbed for that device.

The pulleyboard uses a single Maxon EC40 brushless DC motor and a DES 70/10 motor controller (Maxon Motors). The end effector is a rotational link with nominal moment of inertia $3.63 \cdot 10^{-4} \mathrm{kgm}^{2}$ about the axis of rotation. The moment of inertia can be varied by additional weight rigidly affixed to the link. The link is cable driven with a cable run from the motor to the link and back for a total cable length of $1.58 \mathrm{~m}$. The stainless steel cable goes through a total of four idler pulleys with a diameter of $15 \mathrm{~mm}$ and eight idler pulleys with a diameter of $7.5 \mathrm{~mm}$, in addition to the motor and link shafts with a diameter of $14 \mathrm{~mm}$. This cable route is similar to the second axis of the surgical robot. Also, a force gauge with an additional $15 \mathrm{~mm}$ pulley is included in one part of the cable run to measure cable tension.

On this simplified testbed it is easy to have two shaft encoders, one colocated at the motor and another noncolocated

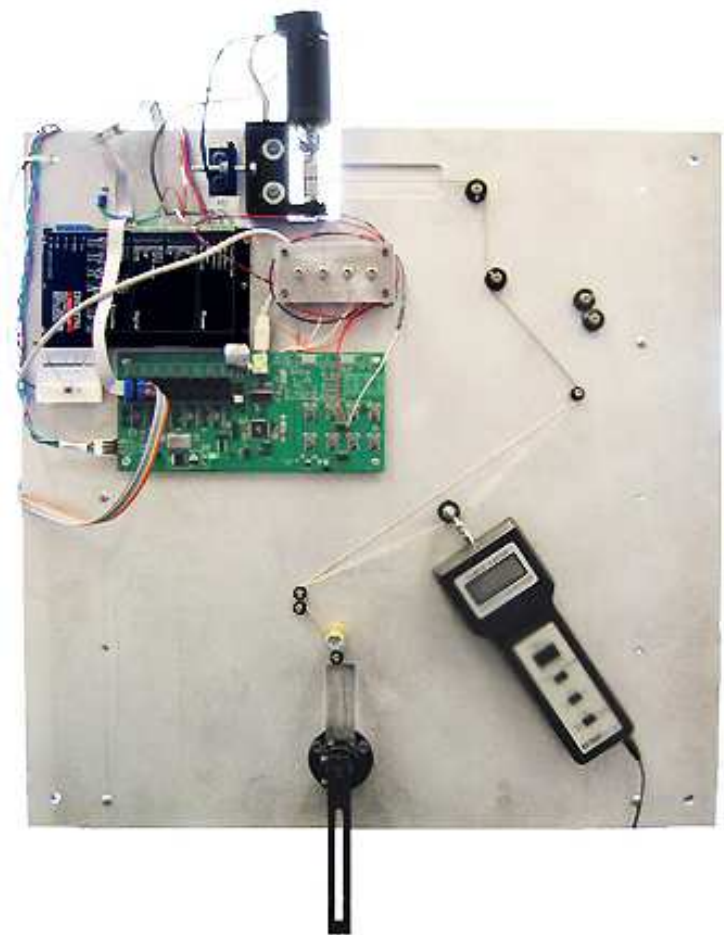

Fig. 1. The pulleyboard with a force gauge in the lower right corner.

at the link. These sensors directly measure motor angle $q_{m}$ and link angle $q_{l}$ (see Fig. 2). So that the results will be applicable to the RAVEN robot, the noncolocated sensor was used only for validation, and not for control or parameter estimation.

Also, identical to the RAVEN the pulleyboard includes an RTAI Linux PC. The PC is a 32-bit AMD Athlon XP with 1GB RAM. I/O with the the pulleyboard uses the BRL USB board developed in the BioRobotics Lab at the University of Washington [14]. The robot control software is an RTAI-Linux kernel module, and the control loop runs at $1 \mathrm{kHz}$.

\section{B. Modeling}

The main modeling assumption is that, although the pulleyboard has several idler pulleys, it can be modeled as one motor side and one link side connected by one cable run. Idler pulley inertias and transmission friction can be lumped into either one of the two sides without any significant loss of accuracy.

Fig. 2 shows a schematic drawing of the pulleyboard the way it is modeled. The pulleyboard model comprises a motor inertia, $J_{m}$, and a link inertia, $J_{l}$, connected by two cables each with a longitudinal stiffness $k_{e}$ and damping $b_{e}$. For the purpose of modeling, the two cables are considered as one torsional spring/damper connecting the two inertias, where the total torsional effect is the combination of the longitudinal effect of the two cables. The spring is modeled as an exponential spring, i.e. for a generic displacement $d$ we have

$$
F_{\text {spring }}=k_{e}\left(e^{\alpha d}-1\right) \text {. }
$$

Initial estimation tests were carried out where both $k_{e}$ and $\alpha$ were considered unknown. However, we were unable to 


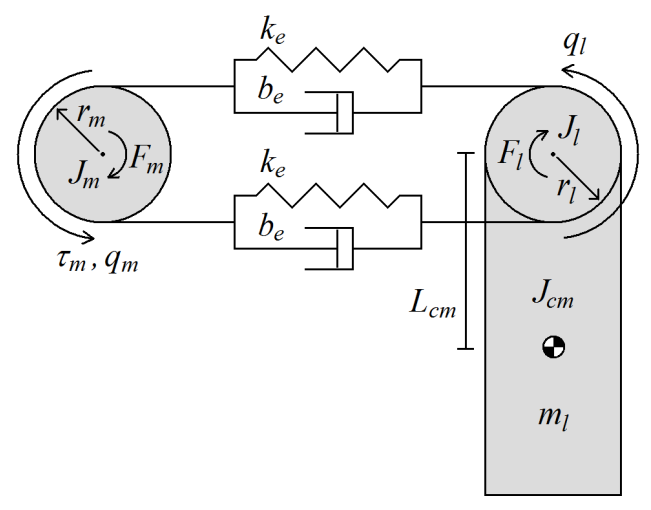

Fig. 2. The pulleyboard model.

make the UKF estimate for $\alpha$ converge. Possibly, this is because the relative displacements of the stiff transmission are very small, and within these displacements a wide range of values for $\alpha$ yield approximately the same force. Instead, $\alpha=1$ throughout this paper. The use of an exponential spring model is nevertheless worthwhile, as the increased degree of nonlinearity will put the UKF further to the test. The damper is modeled as a linear damper. The mass of the cables is neglected. It is assumed that the cables are pretensioned equally, and to a sufficiently high tension that neither cable goes slack under normal operation. Friction in the transmission is lumped into a motor side term $F_{m}$ and a link side term $F_{l}$.

Let $r_{m}=r_{l}$ be the motor and link capstan radii, respectively. Since they are equal the pulleyboard's gear ratio is $1: 1$. Let $\tau_{m}$ be the torque applied to the motor, $q_{m}$ the angle of the motor and $q_{l}$ the angle of the link. The motor and link angles are zero when the link is in a vertical position, the tensions in the two cables are equal and the system is not moving. The dynamic equation is then written as

$$
\boldsymbol{J} \ddot{q}+\boldsymbol{T}+\boldsymbol{F}+\boldsymbol{N}=\boldsymbol{\tau}
$$

where $\boldsymbol{q}=\left[q_{m}, q_{l}\right]^{T}, \boldsymbol{\tau}=\left[\tau_{m}, 0\right]^{T}$, and

$$
\begin{aligned}
& \boldsymbol{J}=\left[\begin{array}{cc}
J_{m} & 0 \\
0 & J_{l}
\end{array}\right], \quad \boldsymbol{T}=\left[\begin{array}{c}
r_{m} \gamma \\
-r_{l} \gamma
\end{array}\right], \\
& \boldsymbol{F}=\left[\begin{array}{c}
F_{m} \\
F_{l}
\end{array}\right] \quad \text { and } \quad \boldsymbol{N}=\left[\begin{array}{c}
0 \\
m_{l} L_{c m} g \sin \left(q_{l}\right)
\end{array}\right] \text {. }
\end{aligned}
$$

The motor inertia $J_{m}$ can be found by consulting the motor's data sheet, and the link inertia $J_{l}$ can be calculated using the parallel axis theorem. The variable $\gamma$ is used to denote the total longitudinal force of the spring/damper effect of both cables. It is calculated as

$$
\gamma=k_{e}\left(e^{q_{m} r_{m}-q_{l} r_{l}}-e^{q_{l} r_{l}-q_{m} r_{m}}\right)+2 b_{e}\left(\dot{q}_{m} r_{m}-\dot{q}_{l} r_{l}\right) .
$$

The lumped transmission friction $\boldsymbol{F}$ is modeled by the simple Coulomb and viscous combination:

$$
F_{i}=F_{c, i} \operatorname{sign}\left(\dot{q}_{i}\right)+F_{v, i} \dot{q}_{i}, \quad i=m, l
$$

where $F_{c, i}$ and $F_{v, i}$ are the Coulomb and viscous friction constants, respectively. Motor and link shaft friction is also included in these terms. Finally, $N$ contains the gravitational torque acting on the link, with $m_{l}$ being the mass of the link, $L_{c m}$ is the distance from the axis of rotation to the center of mass, and $g$ is the acceleration of gravity.

Some of the parameters in the dynamic equation are known, while some are unknown. In particular, the inertias $J_{m}$ and $J_{l}$ are known, and so are $m_{l}$ and $L_{c m}$, so that the gravitational term $N$ can be computed. The capstan radii $r_{m}$ and $r_{l}$ are also known. On the other hand, the stiffness constant $k_{e}$ and the damping constant $b_{e}$ are unknown. So are the four friction constants $F_{c, i}$ and $F_{v, i}$, for a total of six unknown parameters. These parameters have to be estimated.

\section{Methods}

The Unscented Kalman Filter (UKF) was used in two ways: offline for simultaneous state and parameter estimation and online for state estimation. System parameters identified offline were used online for state-only estimation. Although the pulleyboard has motor and link angle available, only motor angle was used so as to emulate a system with colocated sensors and actuators.

A PD controller was implemented for all data collection, both during the offline estimation and the following robustness experiments. The pulleyboard performed sinusoidal trajectory following tasks, and except for the force disturbance tests the pulleyboard was operated in free-space motion. As the topic of our study was state estimation performance, and not control performance, the state estimate was not used for feedback control. Instead, the motor shaft encoder information was used directly for PD control. A low-pass filter was applied to avoid the resonant high-frequency modes inherent in the elastic cable transmission, and velocity was calculated by a first-difference of the low-pass filtered position samples.

\section{A. Offline State and Parameter Estimation}

The key to parameter estimation with the UKF is to regard the unknown parameters as part of the state. That way the basic UKF algorithm does not have to be modified. The pulleyboard's dynamic equation (1) is written in state-space form, discretized, and the state vector is augmented with the unknown parameters.

To write the dynamic equation in state-space form we first define the state vector of the continuous-time system $\boldsymbol{x}(t)$ as

$$
\boldsymbol{x}:=\left[\begin{array}{llll}
q_{m} & q_{l} & \dot{q}_{m} & \dot{q}_{l}
\end{array}\right]^{T} .
$$

We also define the generic input signal $u:=\tau$. The pulleyboard's dynamics in state-space form are then given as

$$
\begin{aligned}
\dot{\boldsymbol{x}} & =\boldsymbol{f}(\boldsymbol{x}, \boldsymbol{u}), \\
y & =\boldsymbol{C} \boldsymbol{x}
\end{aligned}
$$

where

$$
\begin{aligned}
\boldsymbol{f} & :=\left[\begin{array}{c}
\dot{\boldsymbol{q}} \\
\boldsymbol{J}^{-1}(\boldsymbol{\tau}-\boldsymbol{N}-\boldsymbol{F}-\boldsymbol{T})
\end{array}\right], \\
\boldsymbol{C} & :=\left[\begin{array}{llll}
1 & 0 & 0 & 0
\end{array}\right] .
\end{aligned}
$$

The measurement $y$ is equal to motor angle $q_{m}=x_{1}$. 
Since $f$ is nonlinear we cannot compute the exact discretetime equivalent of the continuous-time system. Instead we numerically integrate the state-space equations using a fourth order Runga-Kutta method. Denoting the Runga-Kutta 4 operator by $\boldsymbol{F}_{R K 4}$ we get

$$
\begin{aligned}
\boldsymbol{x}(k+1) & =\boldsymbol{F}_{R K 4}(\boldsymbol{f}(\boldsymbol{x}(k), \boldsymbol{u}(k)))=: \boldsymbol{F}(\boldsymbol{x}(k), \boldsymbol{u}(k)), \\
y(k) & =\boldsymbol{C} \boldsymbol{x}(k)
\end{aligned}
$$

where $k$ is the time index. The measurement equations (3) and (5) for the continuous and discrete-time systems are the same.

The final step before applying the UKF algorithm is to construct the augmented dynamic equation of the pulleyboard. We define the augmented state vector as

$$
\boldsymbol{x}^{a}:=\left[\begin{array}{lllllll}
\boldsymbol{x}^{T} & k_{e} & b_{e} & F_{c, m} & F_{c, l} & F_{v, m} & F_{v, l}
\end{array}\right]^{T} .
$$

The augmented system is then described by

$$
\begin{aligned}
\boldsymbol{x}^{a}(k+1) & =\boldsymbol{F}^{a}\left(\boldsymbol{x}^{a}(k), \boldsymbol{u}(k)\right), \\
y(k) & =\boldsymbol{C}^{a} \boldsymbol{x}^{a}(k) .
\end{aligned}
$$

where

$$
\begin{aligned}
\boldsymbol{F}^{a}\left(\boldsymbol{x}^{a}(k), \boldsymbol{u}(k)\right) & :=\left[\begin{array}{c}
\boldsymbol{F}(\boldsymbol{x}(k), \boldsymbol{u}(k)) \\
k_{e}(k) \\
b_{e}(k) \\
F_{c, m}(k) \\
F_{c, l}(k) \\
F_{v, m}(k) \\
F_{v, l}(k)
\end{array}\right], \\
\boldsymbol{C}^{a} & :=\left[\begin{array}{ll}
\boldsymbol{C} & \mathbf{0}_{1 \times 6}
\end{array}\right] .
\end{aligned}
$$

With the system (6), (7) we are ready for direct application of the UKF algorithm. Essentially, the UKF uses a deterministic sampling approach to calculate the state estimate $\hat{\boldsymbol{x}}^{a}$ and covariance. A set of samples are chosen that completely capture the true mean and covariance of the state $\boldsymbol{x}^{a}$. These samples are called sigma points and they are propagated through the nonlinearity $\boldsymbol{F}^{a}$ instead of the state itself. The state estimate and covariance are then found by weighted average computation. For the offline state and parameter estimation a square-root implementation of the UKF algorithm is used to shorten the execution time [9]. Although process noise $\boldsymbol{v}(k)$ and measurement noise $n(k)$ are not included in (6) and (7) the knowledge of the $10 \times 10$ process noise covariance matrix $\boldsymbol{R}_{v}$ and the measurement noise covariance $R_{n}$ is required. In this paper these are used solely to control the convergence properties of the algorithm. For further details on the derivation and use of the UKF, see e.g. [7, 8, 9].

Before data collection, the six unknown parameters were divided into two groups; one group consisted of the cable parameters $k_{e}$ and $b_{e}$, while the other group consisted of the friction parameters $F_{c, m}, F_{c, l}, F_{v, m}$ and $F_{v, l}$. A separate data set was collected for each group, both with a duration of 140 seconds. For the cable parameters a data set with high frequency motion was used, because it maximizes the relative motion between motor and link side, and thereby provides the most information to the UKF. Also, with respect to the total torque acting on the joints, friction parameters are usually less dominant at high velocities, so they were assumed negligible. The desired motor angle $q_{m}^{d}$ given to the PD controller was

$$
\begin{aligned}
q_{m}^{d}(t)= & \sin (2 \pi t)-0.7 \sin (3 \pi t)+0.5 \sin (4 \pi t) \\
& +0.4 \sin (5 \pi t)-0.2 \sin (6 \pi t)+0.1 \sin (7 \pi t) .
\end{aligned}
$$

For the friction parameters a data set with low frequency motion was used, since friction is a dominant term at low velocities. The low frequency angular trajectory was identical to the high frequency trajectory with frequencies divided by three. The cable parameters found with the first data set were used during this second round of estimation.

In the end all six parameters were estimated. For the online estimation and robustness tests these parameters were considered known and fixed.

\section{B. Online State Estimation Under Nominal Conditions}

Online state estimation was initially tested under nominal conditions to check the baseline state estimation performance, and to validate the parameters found offline. For state-only estimation there is no need to define the augmented system (6), (7), the original system (4), (5) can be used as is. Therefore state-only estimation is easier to implement than combined state and parameter estimation, and the smaller system dimension makes execution faster. Thus, a square-root implementation of the online state estimator was not deemed necessary. As before, the $4 \times 4$ process noise covariance matrix $\boldsymbol{R}_{v}$ and the measurement noise covariance $R_{n}$ were only set to control the convergence properties of the UKF, and did not reflect the real noise in the system. The trajectory used for validation was

$$
\begin{aligned}
q_{m}^{d}(t) & =0.75 \sin (1.6 \pi t)-0.63 \sin (2.6 \pi t) \\
& +0.225 \sin (3.66 \pi t)+0.15 \sin (4.4 \pi t) \\
& -0.12 \sin (6.6 \pi t)+0.075 \sin (6.9 \pi t) .
\end{aligned}
$$

Data were collected for 60 seconds.

The main reason not to adaptively estimate the unknown parameters online is that contact force estimates would be adversely affected. If the end effector were in contact with the environment an adaptive UKF would alter the system parameters to reflect the coupled system's (pulleyboard+environment) behavior. Hence, wrong parameter estimates would be used for contact force estimation.

\section{Robustness Experiments}

The main purpose of the present work is to study the robustness of the Unscented Kalman Filter to changes in system parameters. Robustness was measured in terms of the ability of the UKF to maintain satisfactory real-time state estimation using system parameters identified offline even as the system undergoes dynamic changes. The robustness of the UKF was studied when

- the link inertia was increased from its nominal value,

- the cable tension varied around its nominal value, 
- a contact force on the link increased from its nominal value of zero, and

- the motion of the link was physically constrained.

For each test the motor and link angles were recorded together with the estimated state and input motor torque. Actual velocities were computed during post processing using a zero-phase low-pass filter on the first differences of the encoder signals. The relative root-mean-square (RMS) error and maximum error between actual and estimated state was computed for each test.

1) Link Inertia: When a robot picks up an object the effective inertia changes, which impacts the system dynamics. Also, the inertia matrix of a multi-DoF robot is not constant, as is the case for the 1-DOF pulleyboard, but varies with the robot's configuration. To simplify the system dimension and reduce computing time, it may be desirable to use a 1-DoF UKF for each joint of a multi-DoF robot. For these reasons the UKF must be robust to changes in inertia.

For this experiment the link inertia was changed by attaching weights to the link at several distances from the axis of rotation. Inertia was increased from the nominal link inertia of $3.63 \cdot 10^{-4} \mathrm{kgm}^{2}$ up to $1.33 \cdot 10^{-3} \mathrm{kgm}^{2}$, an increase by a factor of 3.65. For practical reasons it was impossible to decrease the inertia. At each inertia the same sum-of-sinusoids angular trajectory was used as for the validation in section III-B.

2) Cable Tension: The amount of pretension in the cable indirectly determines the cable's spring and damping parameters, and also the lumped friction parameters. Since the cable tension will change over time, so will the parameters that depend on it. By varying the tension around the nominal value used for parameter estimation we measured the robustness of the UKF to changes in estimated parameters.

Tension was varied in steps of approximately $260 \mathrm{~g}$ from the maximum tension of $3.3 \mathrm{~kg}$, via the nominal tension of $2.2 \mathrm{~kg}$, down to $1.17 \mathrm{~kg}$. The tensions tested ranged from about 0.5 to 1.5 times the nominal tension. Again, the same sinusoid was used as for the validation in section III-B.

3) Contact Force: Contact force estimation is of interest in teleoperation as a potential replacement for force sensors. The contact force can be computed with complete knowledge of the input torque, system state and dynamics. However, it is often the case that the complete state is not known, and it is therefore desirable to see how the state estimates are affected as a result of the applied contact force.

Accurate contact forces were simulated by attaching a cable perpendicular to the link and pulling with weights in a hanging basket. Meanwhile the pulleyboard followed the desired trajectory:

$$
q_{m}^{d}(t)=0.5 \sin (\pi t) .
$$

A single $0.5 \mathrm{~Hz}$ sine wave was used instead of (8) to keep the basket from jumping around. The quasi-static conditions ensured that the additional inertia was not affecting the dynamics, and that the weight was simply a constant force. At first the contact force corresponded to the weight of the basket only (12.5 g), and then weights of $12.5 \mathrm{~g}$ were
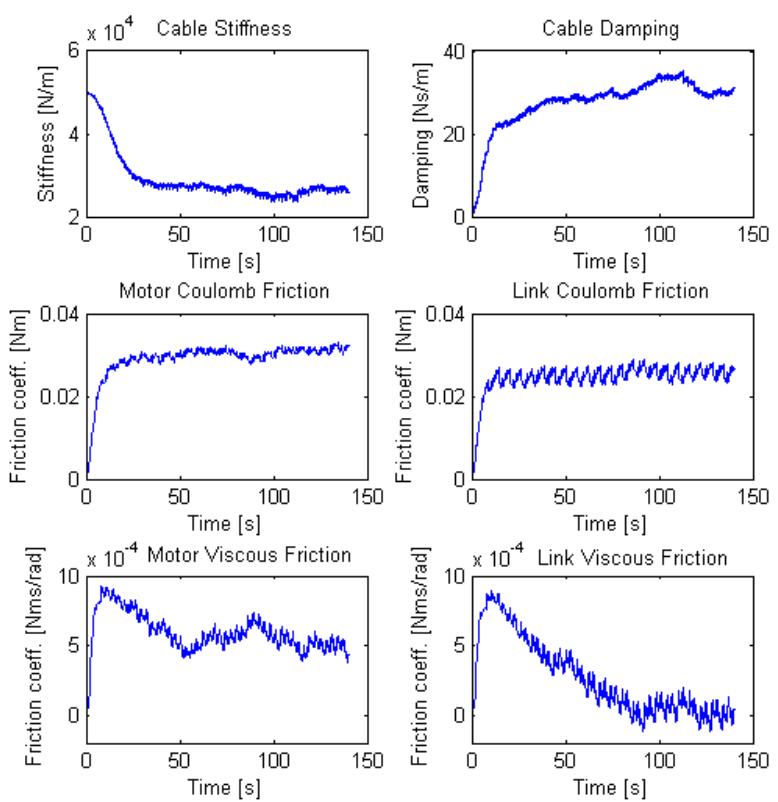

Fig. 3. Offline convergence of the six estimated parameters

added every 20 seconds. Weights were added 7 times for a total of 8 data points. The maximum contact force was $8 \times 12.5 \mathrm{~g} \times 9.81 \mathrm{~m} / \mathrm{s}^{2}=0.981 \mathrm{~N}$. Since there was a small transient when new weights were added only the data in the middle 10 seconds of every 20-second interval were used.

4) Constrained Motion: When the link of the pulleyboard is physically constrained, a worst case scenario is created wherein the UKF assumes the robot is moving when it is not. Due to the constraint, the link will not move at all, but the motor will because of the elastic transmission. Since the cable transmission is very stiff $\left(k_{e}=3 \cdot 10^{4} \pm 1 \cdot 10^{4} \mathrm{~N} / \mathrm{m}\right)$, the difference between motor angle and link angle will always be small (typically $<0.15 \mathrm{rad}$ ). Hence, the UKF state estimator must be very accurate in order to provide any additional information about the actual link state. The alternative would be to assume that the motor and link states are equal. We can get a clear picture of whether the implementation of the UKF is worthwhile if the UKF link state estimate is better than using the colocated sensor to approximate the link state.

To check this, the pulleyboard was operated with the link physically constrained for 20 seconds. The link was rigidly fixed to the base using a C-clamp, and a sinusoidal trajectory of $0.5 \mathrm{~Hz}$ was commanded to the PD controller.

\section{RESULTS}

\section{A. Offline State and Parameter Estimation}

The results of the offline parameter estimation are shown in Fig. 3. The top two graphs show the convergence of the spring and damper constants, which were found with the high frequency data set. The bottom four graphs show the friction terms, which were found with the low frequency data set. All six of the estimated parameters showed convergence. For this data set, the Coulomb friction tended to converge quickly in 

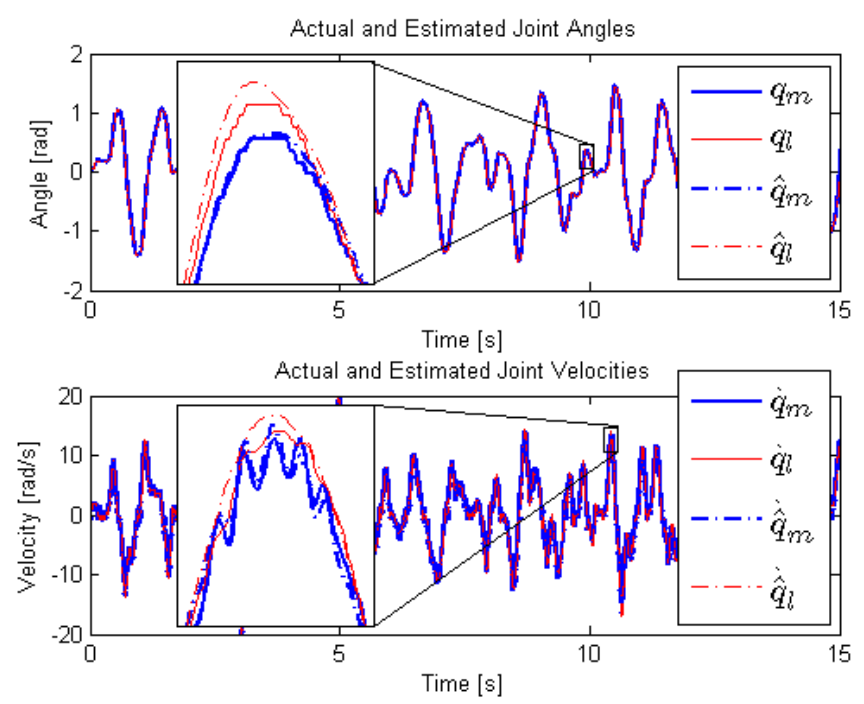

Fig. 4. Online state estimation under nominal conditions: 15-second excerpt and zoom detail.

10-20 seconds, while the stiffness and damping parameters showed slower convergence. The viscous friction took even longer to converge; the link viscous friction in particular took nearly 100 seconds to reach steady state.

\section{B. Online State Estimation Under Nominal Conditions}

Fig. 4 shows a 15 -second excerpt from the 60 -second data set, plus a detail at high zoom level. The state estimates are denoted by $\hat{q}_{m}, \hat{q}_{l}, \dot{\hat{q}}_{m}$ and $\dot{\hat{q}}_{l}$. At high magnification the difference between motor and link angles becomes evident, whereas the difference in velocity is less pronounced. The zoom window in the lower plot shows oscillations in the actual motor velocity, and the state estimator tracking those oscillations in phase. The link velocity is less oscillatory, and the link velocity estimate reflects that.

\section{Robustness Experiments}

Table I contains relative RMS estimation errors in percent for nominal and off-nominal experiments, calculated as

$\mathrm{RMS} \%=100 * \operatorname{RMS}($ estimation error) $/ \mathrm{RMS}$ (actual value).

Absolute errors are used for constrained motion. The errors are $\tilde{q}_{m}:=q_{m}-\hat{q}_{m}, \tilde{q}_{l}:=q_{l}-\hat{q}_{l}, \dot{\tilde{q}}_{m}:=\dot{q}_{m}-\dot{\hat{q}}_{m}$ and $\dot{\tilde{q}}_{l}:=\dot{q}_{l}-\dot{\hat{q}}_{l}$.

1) Link Inertia: Fig. 5 shows the relative RMS error and the maximum error of the state estimation as link inertia is increased. All four graphs show the same phenomenon; the error stays almost constant until the inertia reaches 2.5 times its nominal inertia. It is then much higher for an inertia of 3.5 times nominal, and especially pronounced for the link angle.

2) Cable Tension: Fig. 6 shows the relative RMS and maximum values of the state estimation error as the cable tension varies around its nominal value. The motor angle estimation error stays relatively constant. Link angle error, however, is lower than nominal for lower tension, and gets worse with higher tensions. The RMS error of the velocity estimates stays flat up to the nominal tension, and then
TABLE I

RELATIVE RMS ERRORS (\%) FOR ROBUSTNESS EXPERIMENTS. FOR COMPARISON, THE RELATIVE RMS ERROR OF MOTOR ANGLE TO LINK ANGLE IS INCLUDED. ${ }^{\dagger}$ THE LAST ROW CONTAINS ABSOLUTE RMS ERROR VALUES IN RAD OR RAD/S

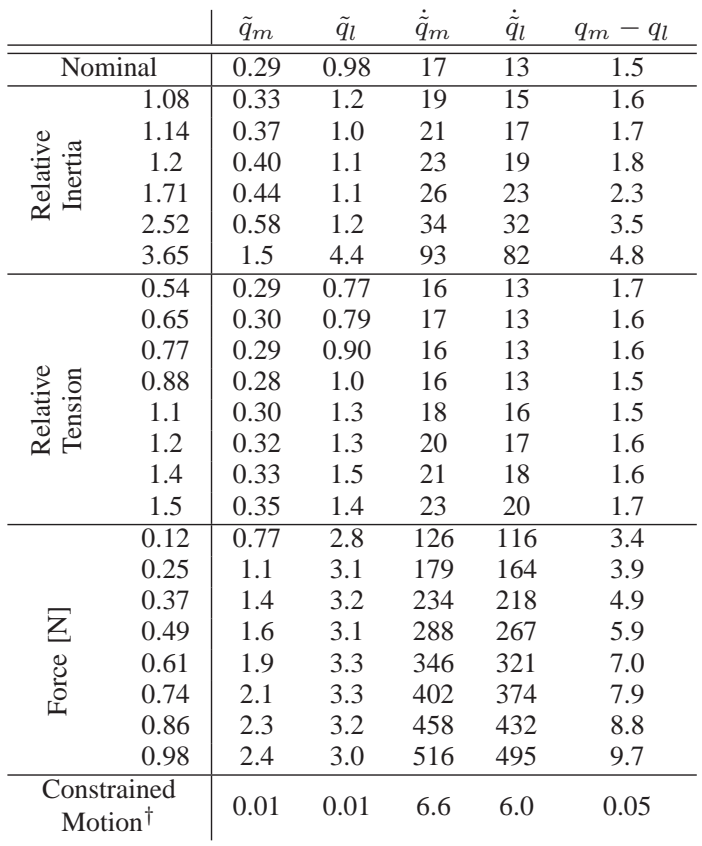
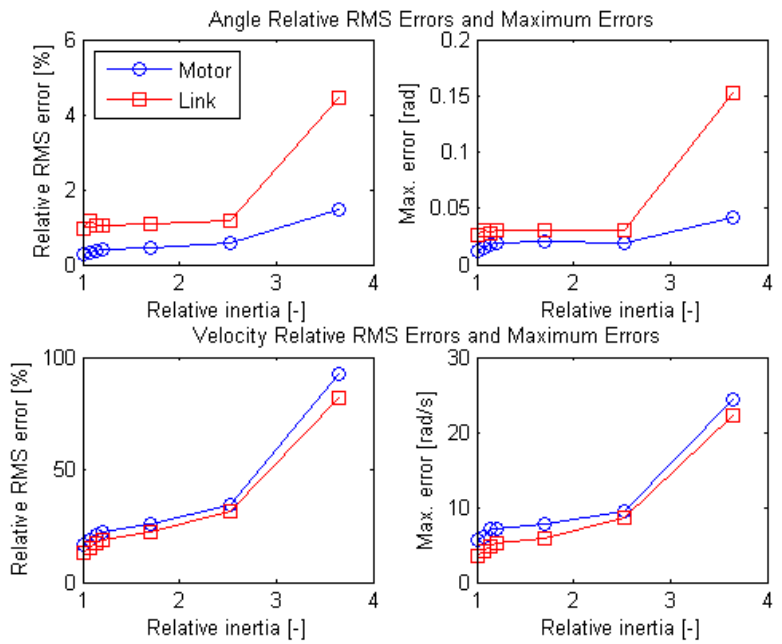

Fig. 5. State estimation performance expressed in terms of relative RMS error and maximum error for increasing link inertia.

increases almost linearly with higher tension. It is hard to find a consistent pattern in the maximum errors. However, the link angle error does seem to go up as tension increases.

3) Contact Force: Fig. 7 shows the effect of constant applied force on estimation error. Motor angle and velocity, and link velocity all have errors with near-linear characteristics, whereas the link angle estimate has a somewhat more random shape and shows less variation. It should be noted that velocity estimation performance is very poor. 

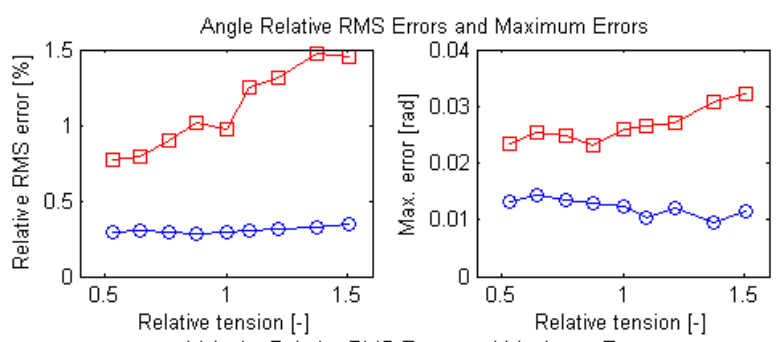

Velocity Relative RMS Errors and Maximum Errors
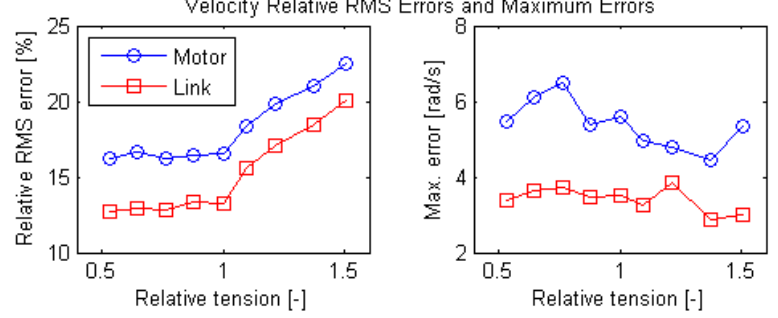

Fig. 6. State estimation performance expressed in terms of relative RMS error and maximum error for changing cable tension.
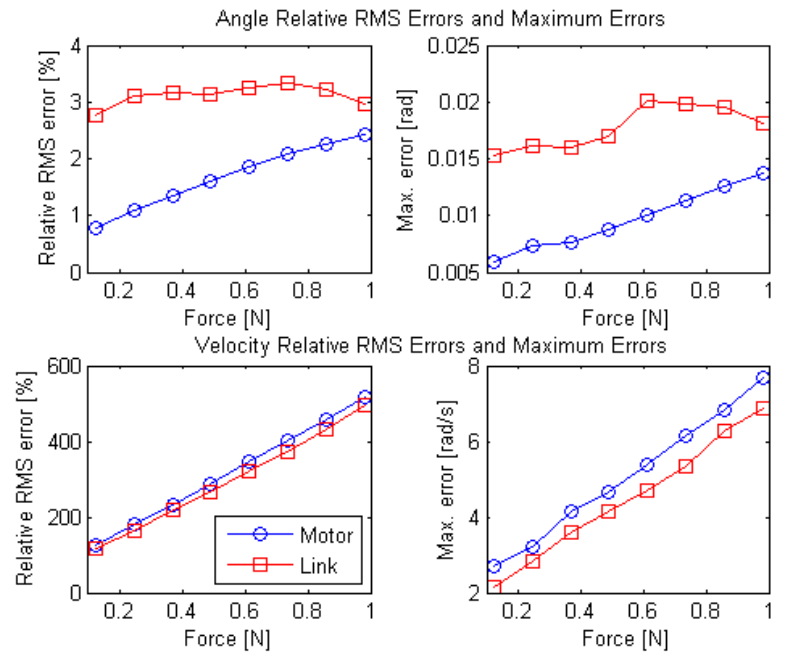

Fig. 7. State estimation performance expressed in terms of relative RMS error and maximum error for increasing contact force.

4) Constrained Motion: Fig. 8 shows the system response to an input of 0.5 radians at $0.5 \mathrm{~Hz}$ under constrained motion. The motor angle estimate is still close to the actual angle, and the link angle estimate is smaller than the motor angle, closer to the real link angle. The link angle appears to be out of phase with the motor trajectory tracking. Both velocity estimates are far away from the actual values, at or near zero.

\section{DISCUSSION}

Offline parameter estimation yielded reasonable values for all six of the desired parameters. Dividing the parameters into two groups and estimating them separately was necessary to have all parameters converge to good estimates. Also, the choice of initial value for the cable stiffness was important for the convergence outcome. Setting the initial value of $k_{e}$ to zero did not lead to convergence. In our case the initial stiffness guess was set at $5 \cdot 10^{4} \mathrm{~N} / \mathrm{m}$. It is interesting to note that the
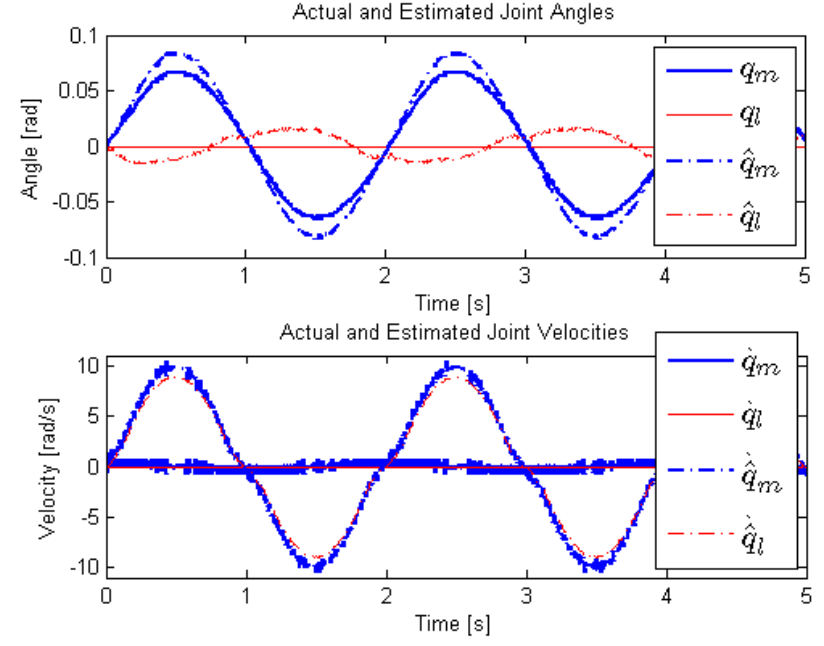

Fig. 8. State estimation when link is physically constrained.

results showed Coulomb friction to be greater than viscous friction by about two orders of magnitude. This agrees with findings in [15], where it was found that pulley friction was primarily Coulombic. The assumption made in section III-A that friction is less dominant at higher velocities also hinged on this observation.

In the nominal online tests motor and link states were accurately estimated using the system parameters found in the offline estimation step. Velocity estimation was not as good as angle estimation. However, the velocity estimate was still significantly improved from the regular low-pass filtered first difference and, in particular, there was no phase lag in the UKF velocity estimate. Phase lag is a common factor in controller instability and poor controller performance. With the improved estimates we will be able to increase the controller gains and improve tracking performance.

The right-most column in Table I contains the relative RMS values of $q_{m}-q_{l}$. This is the error in using motor angle as a substitute for link angle. The RMS error of the motor and link angle estimation $\tilde{q}_{m}$ and $\tilde{q}_{l}$ were consistently smaller showing that performance of the UKF was satisfactory and derived more useful information. This result is also visible in Fig. 4. We therefore conclude that the UKF is worthwhile for estimating link state from colocated sensors. Note that the RMS error shot up when the inertia of the link was increased from a factor of 2.5 to 3.6. This suggests a "safe range" of inertial changes, or weights that the robot can hold without affecting state estimation too much. Also, care should be taken in using the velocity estimate during environmental contact.

Fig. 7 reveals a near-linear relationship between contact force and estimation error for all states except link angle. The reverse relationship would suggest using error to estimate contact force, especially from the motor angle estimate, since it is measurable in real time. A similar technique was also proposed in [16]. However, we would only feel confident doing this under quasi-static conditions, because that is how the experiments were carried out. 
Overall, angle estimates were more robust to changes in dynamics than velocity estimates. This is especially true for motor angle estimates. That is to be expected, as motor angle is closest to the sensor used to compute the estimates (only separated by quantization). Thus, if only angle information is used in controllers or for further estimation, the UKF may be used to achieve desired robustness in changes to the operating conditions. Although velocity estimates were less robust, phase information was kept intact, at least during the inertia and tension experiments. In feedback control the phase of the velocity is more important than its amplitude, because phase lag is a common source of instability. Hence, our velocity estimates may still be used in feedback loops over some range of operating conditions, at least in free-space motion.

In all Figs. 5-7 motor angle estimation errors were smaller than link errors, while the opposite was true for the velocities. We believe that the reason why the motor angle estimates were better than their link counterparts is simply that the encoder was located on the motor side (remember that the link side encoder was only used for validation). Furthermore, we believe that the link velocity estimation errors were smaller because the motor velocity signal contained more high frequency oscillations making it harder to predict.

Observing the RMS error versus changes in inertia, tension or contact force, we expect the minimum error at the nominal conditions. For the inertia and contact force tests this was the case, but not for the tension tests. Fig. 6 shows that instead it seems that RMS values decreased slightly for smaller tensions, while increasing almost linearly with tensions above nominal. This suggests that when cable tension is reduced due to cable stretch or other factors, state estimate will not be adversely affected. Overall, change in error due to unmodeled changes in tension were relatively small, meaning UKF performance is quite robust in that respect. This is important, since cable tension is bound to change with time in a cable-driven robot.

\section{CONClusion \& Future Work}

The Unscented Kalman Filter has been tested for state and parameter estimation in an elastic transmission using only motor angle measurements. The performance and robustness of the UKF to changes in system dynamics has been studied.

During normal operation the UKF was able to distinguish between motor and link angles although the transmission was very stiff, and it provided additional information about the actual link state that could not be measured directly with the motor encoder. This is useful in the context of state feedback in control loops.

Robustness experiments showed that angle estimates (motor and link) were robust to changes in operating conditions, such as contact with the surroundings or variation in dynamic parameters. Velocity estimates were less robust.

An almost linear relationship between increasing contact force and estimation errors suggested using the errors for estimating the contact force.

Future work will mainly focus on implementing the UKF for state and parameter estimation on the RAVEN robot. While our results show that under a range of conditions a joint level UKF can provide better state estimation than is possible without such a filter, implementation on a multi-DoF system will undoubtedly bring new challenges and discoveries. We also intend to study the importance of nonlinear versus linear models for the transmission elasticity.

\section{ACKNOWLEDGEMENT}

This work was partially funded by The Research Council of Norway project 167529/V30.

\section{REFERENCES}

[1] M. J. H. Lum, D. Friedman, J. Rosen, G. Sankaranarayanan, H. H. King, K. Fodero, R. Leuschke, M. Sinanan, and B. Hannaford, "The RAVEN - design and validation of a telesurgery system," International Journal of Robotics Research, May 2009.

[2] S. Nicosia and A. Tornambé, "A new method for the parameter estimation of elastic robots," in Proc. IEEE International Conference on Systems, Man and Cybernetics, vol. 1, Beijing, China, Aug. 1988, pp. 357-360.

[3] S. Nicosia, P. Tomei, and A. Tornambé, "A nonlinear observer for elastic robots," IEEE Trans. Robot. Automat., vol. 4, no. 1, pp. 45-52, Feb. 1988.

[4] M. Jankovic, "Observer based control for elastic joint robots," IEEE Trans. Robot. Automat., vol. 11, no. 4, pp. 618-623, Aug. 1995.

[5] N. Léchevin and P. Sicard, "Observer design for flexible joint manipulators with parameter uncertainties," in Proc. IEEE International Conference on Robotics and Automation, vol. 3, Albuquerque, NM, USA, Apr. 1997, pp. 2547-2552.

[6] F. Abdollahi, H. A. Talebi, and R. V. Patel, "A stable neural networkbased observer with application to flexible-joint manipulators," IEEE Trans. Neural Networks, vol. 17, no. 1, pp. 118-129, Jan. 2006.

[7] S. J. Julier and J. K. Uhlmann, "A new extension of the Kalman filter to nonlinear systems," in Proc. SPIE 11th International Symposium on Aerospace/Defense Sensing, Simulation and Controls, vol. 3068, Orlando, FL, USA, Apr. 1997, pp. 182-193.

[8] E. A. Wan and R. var der Merwe, "The unscented Kalman filter for nonlinear estimation," in Proc. IEEE Symposium on Adaptive Systems for Signal Processing, Communications and Control, Lake Louise, Canada, Oct. 2000, pp. 153-158.

[9] R. van der Merwe and E. A. Wan, "The square-root unscented Kalman filter for state and parameter estimation," in Proc. IEEE International Conference on Acoustics, Speech and Signal Processing, vol. 6, Salt Lake City, UT, USA, May 2001, pp. 3461-3464.

[10] N. Araki, M. Okada, and Y. Konishi, "Parameter identification and swing-up control of an acrobot system," in Proc. IEEE International Conference on Industrial Technology, Hong Kong, China, Dec. 2005, pp. $1040-1045$.

[11] X. Zhao, J. Lu, W. P. A. Putranto, and T. Yahagi, "Nonlinear time series prediction using wavelet networks with Kalman filter based algorithm," in Proc. IEEE International Conference on Industrial Technology, Hong Kong, China, Dec. 2005, pp. 1226-1230.

[12] J. H. Gove and D. Y. Hollinger, "Application of a dual unscented Kalman filter for simultaneous state and parameter estimation in problems of surface-atmosphere exchange," Journal of Geophysical Research, vol. 111, Apr. 2006.

[13] R. Zhan and J. Wan, "Neural network-aided adaptive unscented Kalman filter for nonlinear state estimation," IEEE Signal Processing Letters, vol. 13, no. 7, pp. 445-448, July 2006.

[14] K. Fodero, H. H. King, M. J. H. Lum, C. Bland, J. Rosen, M. Sinanan, and B. Hannaford, "Control system architecture for a minimally invasive surgical robot," Medicine Meets Virtual Reality 14: Accelerating Change in Healthcare: Next Medical Toolkit, 2006.

[15] C. R. Johnstun and C. C. Smith, "Modeling and design of a mechanical tendon actuation system," Journal of Dynamic Systems, Measurement and Control, vol. 114, no. 2, pp. 253-261, June 1992.

[16] P. J. Hacksel and S. E. Salcudean, "Estimation of environment forces and rigid-body velocities using observers," in Proc. IEEE International Conference on Robotics and Automation, San Diego, CA, USA, May 1994, pp. 931-936. 